\title{
MedienPädagogik
}

Zeitschrift für Theorie und Praxis der Medienbildung

Themenheft Nr. 29: Die Konstitution der Medienpädagogik. Zwischen interdisziplinärem Forschungsfeld und bildungswissenschaftlicher (Sub-) Disziplin.

Hrsg. v. Christian Swertz, Wolfgang B. Ruge, Alexander Schmölz und Alessandro Barberi.

\section{Medienbildung - Konstitutionsversuche in der allgemeinen Erziehungswissenschaft Begriffliche Auslotungen eines zeitgenössischen Phänomens}

Caroline Grabensteiner

\begin{abstract}
Zusammenfassung
In der Diskussion um Medienbildung und Medienkompetenz hat sich eine Riege an Autoren etabliert, die innerhalb der Allgemeinen Erziehungswissenschaft den Diskurs um grundlegende Begrifflichkeiten und Fragestellungen am Leben halten und hielten. Der vorliegende Artikel präsentiert die Ergebnisse einer Analyse, die sich dezidiert mit den Positionen von Dieter Spanhel, Norbert Meder, Winfried Marotzki und Werner Sesink auseinandersetzte, um deren Beitrag zur Konstitution der Medienpädagogik als eigenständiger Teildisziplin der Erziehungswissenschaft zu untersuchen. Als zentrale Begriffe wurden in der Untersuchung «Bildung» und «Medium/Medien» gewählt, sowie «Spezifika von Medienbildung» in Abgrenzung oder Vereinnahmung der jeweiligen Bildungsbegriffe. Es handelt sich dabei um eine Untersuchung der Beiträge der vier Autoren zur Debatte um die Definition relevanter Phänomene einerseits und andererseits um die immer wieder neue Aushandlung der Begriffe im Sinne lebhafter Wissenschaft. Ziel der Arbeit ist die Analyse der Verbindung zwischen den Begrifflichkeiten «Medien» und «Bildung» zur «Medienbildung» und der darin auffindbaren argumentativen und begrifflichen Strukturen.
\end{abstract}

Medienbildung - Attempts of constituting within the philosophy of education

\begin{abstract}
This article intends the presentation of the results of an analysis, discussing four positions in the discourse regarding the phenomenon of «Medienbildung». To this purpose the theories of Dieter Spanhel, Norbert Meder, Winfried Marotzki and Werner Sesink are compared concerning the idioms that are used in the debate about media education and defining «Bildung», "Media» and the specifics of «Medienbildung». The four educational scientists and philosophers have contributed to the lively debate in defining the phenomenon and in keeping alive the discourse about its foundation and central definitions. All four authors refer to the modern discourse about information technology and its impact on and consequences for education in theory and practice. The attempt is to find out how the modern discussion refers to classical theories of «Bildung» as well as modern media theories and in what ways the authors argue this connection.
\end{abstract}




\section{Anbindungen}

Der Diskurs um Medienbildung ist angesichts der Entwicklungen moderner Kommunikations- und Informationstechnologie und der damit einher gehenden Beteiligung nicht nur pädagogischer, sondern auch politischer und ökonomischer Akteure zu einem breiten Phänomen geworden. Sich darin dem Aufsuchen «einheimischer Begriffe» (Meder 2007, 55) zu widmen erscheint aufgrund der Materialdichte und Vielfalt der Autoren als ambitioniertes Projekt. Der vorliegende Artikel präsentiert die Ergebnisse einer Analyse, welche sich diesem Vorhaben - zumindest bezogen auf einen Teildiskurs innerhalb der allgemeinen Erziehungswissenschaft - widmete. Dabei geht es weniger um eine Klassifizierung des Medienbildungsbegriffs, wie sie Jörissen vornimmt (Jörissen 2011), sondern um die Beleuchtung einzelner Positionen innerhalb der Medienpädagogik als akademische Disziplin.

Gemeint ist die lebhafte Debatte innerhalb eines Kreises von Erziehungswissenschaftlern, die einerseits in der Allgemeinen Pädagogik und Bildungsphilosophie ihre Wurzeln haben, sich andererseits davon ausgehend mit modernen medialen Phänomenen, wie Computertechnologie und -technik und Internet beschäftigten. Namentlich sind damit Dieter Spanhel, Norbert Meder, Winfried Marotzki und Werner Sesink gemeint ${ }^{1}$. Alle vier Autoren leisten ihren Beitrag zu einer theoretischen Grundlegung der Medienpädagogik von den 80er Jahren bis heute. Besonders die Mitgliedschaft der untersuchten Autoren im sog. "Theorie-Forum» (Fromme und Sesink 2008, 7) und Publikation in und Herausgabe von Formaten, die als Schauplatz der Diskussion um die «grundsätzlichen Theoriediskurse und Reflexionen» (ebd., 8) dienten (s. u. a. Sesink et al. 2007; Fromme und Sesink 2008; Gross et al. 2008; Grell et al. 2010; Fromme et al. 2011; Marotzki und Meder 2014) waren Auswahlkriterien für die Berücksichtigung in der Analyse.

In einem grösseren Zusammenhang gesehen ist dieser Teildiskurs ein Aspekt der Grundsatzdebatte um die Begriffe Medienkompetenz und Medienbildung innerhalb der Medienpädagogik (siehe Zusammenfassend Schorb 2009). Diese entzündete sich im Rahmen der Etablierung der Medienpädagogik als eigenständige wissenschaftliche Disziplin. Es kristallisierten sich zwei Grundbegriffe heraus: «Medienbildung» und «Medienkompetenz». Ein Ausschnitt der lebhaften Debatte und Bemühungen um die Etablierung von Begrifflichkeiten ist Gegenstand der vorliegenden Untersuchung. Besonders interessant ist die Diversität der untersuchten Positionen, welche aufgrund der gemeinsamen - wissenschaftlich-philosophischen - Herkunft und Positionierung der vier Theoretiker in der Kontroverse nicht in dieser Intensität zu erwarten gewesen wäre.

1 Sie alle hatten Lehrstühle der allgemeinen Erziehungswissenschaft inne, widmeten sich der Bildungsphilosophie und insbesondere dem dargestellten Diskurs. Aus diesem Grund wurde im Titel plakativ die allgemeine Erziehungswissenschaft genannt, auch wenn hier einschränkend hinzuzufügen ist, dass diese natürlich ebenso andere Diskurse umfasst. 
Die Auswahl der Autoren erfolgte bewusst aufgrund ihrer Schwerpunkte, die sich als bildungsphilosophische Auslotungen der Wirkungen und Konsequenzen der Entwicklungen moderner Kommunikations- und Informationstechnologien für Bildung grob zusammenfassen liessen. Sie begeben sich in einen Diskurs um die Konstituierung der Disziplin mit eigenständigen Fragestellungen. «Bildung» und ihre Definition ist dabei ein Schlüsselbegriff, seine Definition ist essentiell um das Verständnis von «Medienbildung» im angesprochenen Teildiskurs zu rekonstruieren. Eine Analyse des Diskurses um Medienbildung wird ebenfalls von Jörissen durchgeführt. Seine Untersuchung ist allerdings auf einer breiteren Ebene angesiedelt. Er berücksichtigt den politischen und praktischen Diskurs und stellt ihn neben jenen der qualitativen Bildungsforschung. Er definiert Bildung als «qualitativ-empirisch rekonstruierbare[n] Prozess der Transformation von Selbst- und Weltverhältnissen» (Jörissen 2011, 213). Diese Spezifikation repräsentiert lediglich eine der vier in dieser Arbeit diskutierten Konzepte des Medienbildungsbegriffs, nämlich jene Marotzkis, was der Tatsache geschuldet ist, dass Jörissen und Marotzki eng zusammenarbeiteten².

Bei der systematisch-komparativen Analyse stehen die Begriffe «Bildung» und «Medien» im Fokus. Zur Begriffsanalyse tritt eine Untersuchung der argumentativen Verbindungen zwischen den beiden Begriffen, um letztendlich zur «Medienbildung» als zentralem Gesichtspunkt zu kommen. Dabei kann es nicht das Ziel sein, die Positionen um ihr Eigenrecht zu bringen und aufgrund von Gemeinsamkeiten auf eine daraus resultierende Medienbildungstheorie zu schliessen. Die Analyse führt abschliessend die Engführung auf zentrale Begrifflichkeiten über eine Rekontextualisierung zurück in ihren Bedeutungszusammenhang, um der Vielstimmigkeit des Phänomens Rechnung zu tragen.

\section{Vorgehensweise}

Die Darstellung der vier Theorien, sowie der systematische Vergleich folgten den Ausführungen zur hermeneutischen Methode nach Rittelmeyer und Parmentier (2001, 41). Dabei wurden die elf methodologischen Grunderkenntnisse nach Klafki (2001), berücksichtigt. Vor allem der hermeneutische Zirkel, die Analyse von Begrifflichkeiten und ihrer Verwendung, sowie die Einbeziehung von «Gegenspielern» sind hervorzuheben.

Die Ermittlung der Kriterien erfolgte aus zwei explizierten (Vor-)Annahmen, die sich bei der Beobachtung des Diskurses ergaben. Sie wurden an der Literatur einer Revision unterzogen. Die erste zentrale Prämisse ist, dass die Autoren mit einander im Diskurs stehen und um sich gegenseitig verständlich zu bleiben, die verwendeten Begrifflichkeiten explizieren und zur Disposition stellen. Zweitens wird davon ausgegangen, dass die Argumentationslinien zwischen «Bildung» und «Medium/Medien»

2 U.a. Jörissen und Marotzki 2008, 2009, 2010. 
trotz der unterschiedlichen Stossrichtungen und Herangehensweisen eine Vergleichbarkeit aufweisen. Diese beiden zentralen Hypothesen bilden die Voraussetzung für das Projekt eines systematischen Vergleichs. Die «Frage nach der Bedeutung einzelner Worte und Formen» (Klafki 2001, 138) wird dabei in den Fokus gerückt und zum Leitmotiv der Analyse erhoben. Die Frage danach, wie mit Begrifflichkeiten operiert wird, und welche argumentativen Verbindungen sich dabei auffinden lassen, um im systematischen Vergleich Anhaltspunkte zu haben, wird als zentrales Erkenntnisinteresse ausgewiesen. Im Sinne der Einbeziehung der «jeweiligen Gegenspieler in die Interpretation» (Klafki 2001, 140) könnte in dieser Untersuchung - im gemeinsamen Bemühen der vier Autoren um die Konstitution von Schlüsselbegriffen - auch von «Mitspielern» gesprochen werden. Klafkis Ansicht, dass Texte im pädagogischen Kontext (und nicht nur dort) oft Ausdruck einer bestimmten Position in einem bestimmten streitigen Zusammenhang sind (Klafki 2001, 140), wird grundlegend in die Analyse mit aufgenommen und ist zentrales Merkmal der Auseinandersetzung mit den Texten. Spanhel, Meder, Marotzki und Sesink beziehen jeweils Stellung zu derselben Sache - der theoretischen Grundlegung der Medienbildung aus der Perspektive allgemeiner Erziehungswissenschaft - und erläutern ihre Positionen mittels Publikationen, welche Gegenstand der Analyse sind. Es wird nicht von einzelnen Texten ausgegangen, sondern nach zentralen Aussagen und Schlüsselbegriffen und deren Bedeutungsgehalt in Hinblick auf die nachfolgende Analyse gesucht. Dabei ist die syntaktische Analyse ebenso gegeben, wie der Nachvollzug der «gedankliche[n] Gliederung» (Klafki 2001, 143).

Im Folgenden werden die Ergebnisse der Untersuchung dargestellt, welche sich in die oben beschrieben Begriffe gliedert: Erstens «Medien/Medium» und zweitens «Bildung». Im Sinne des systematischen Vergleichs wurde drittens auf «Verbindungen zwischen Bildung und Medien» eingegangen, um die vereinzelten Begrifflichkeiten wieder in ihre theoretischen Zusammenhänge zu betten und die einzelnen Positionen und die darin entwickelten Spezifika von Medienbildung darzustellen. Im Laufe der Bearbeitung der Quellen wurde es notwendig, diese ursprünglich bewusst offen gehaltenen Kategorien - im Sinne des hermeneutischen Zirkels - durch zusätzliche Fragen zu konkretisieren. Es entwickelten sich dabei mehrere Leitfragen, welche hier zusammengefasst dargestellt werden.

Zu den begrifflichen Kategorien «Medien/Medium» und «Bildung» waren folgende Fragestellungen leitend:

- Welche theoretischen Konzepte werden von den jeweiligen Autoren zur Definition des Medien- und Bildungsbegriffes herangezogen?

- Was sind zentrale Aspekte des Medienbegriffs und des Bildungsbegriffs in den jeweiligen Theorien? 
Zur dritten Kategorie «Verbindungen zwischen Bildung und Medien» ergab sich eine Zweiteilung:

- Erstens wird danach gefragt, wie sich das Verhältnis von Bildung und Medien in den unterschiedlichen Konzeptionen konstituiert und welche Positionen die Autoren dabei einnehmen (also blicken sie aus der pädagogischen Perspektive auf Medien, gehen sie eher analytisch vor, oder positionieren sie sich in der Medientheorie und diskutieren sie deren Einfluss auf pädagogische Fragestellungen, etc.)

- Zweitens interessiert in diesem Zusammenhang auch, welche Spezifika von Medienbildung sich - in Abgrenzung von oder in Vereinnahmung des Bildungsbegriffes - auffinden lassen. Dies wurde wieder mit der Suche nach gemeinsamen Begrifflichkeiten und der Analyse ihrer unterschiedlichen Besetzung in den vier Theorien geleistet.

Die Darstellung der vier Theorien wird im Rahmen dieses Artikels aus Platzgründen verkürzt und in die Präsentation der Ergebnisse eingebettet - auch um Redundanzen zu vermeiden. Es sei dabei angemerkt, dass auch im Zuge der Konzentration auf die Schlüsselbegriffe Bildung, Medien und Medienbildung und die darauf gerichtete Begriffsarbeit schon selektiert wurde.

\section{Ergebnisse}

Als Prolegomenon ist hier zu sagen, dass der deskriptive Zugang ein Mittel ist, die Analyse auf die zentrale Punkte der Theorien zu lenken, ohne die Individualität der einzelnen Positionen zu verschleiern, da die Selektion und zielgerichtete Fragestellung schon interpretierend in den Diskurs eingreift. Es konnte gezeigt werden, dass die unterschiedlichen Ansätze insofern vergleichbar sind, als sie ähnliche theoretische Bezugspunkte aufweisen, was Referenzautoren aber auch analoge Begrifflichkeiten betrifft. Medienbildung lässt sich nach der Untersuchung nicht leichter fassen, dennoch konnten wichtige Gemeinsamkeiten des Diskurses festgestellt und als Schlüsselbegriffe akzentuiert werden.

Im Folgenden wird die Analyse nachgezeichnet, dabei sollen und einzelne Aspekte vertiefend veranschaulicht werden.

\section{Medienbegriff}

Als erster Schlüsselbegriff wurden unterschiedliche Ausprägungen von «Medien», bzw. «Medium» aufgezeigt. Die Analyse gliedert sich in drei Schritte: Erstens «die Medien - das Medium» als Zusammenschau der allgemeinen Definitionen in Anlehnung an die in den Theorien entwickelten Medienbegriffe. Zweitens geht es um «Sprache und Kultur» als mediale Phänomene, die in allen vier Positionen verhandelt werden. 
Drittens wird via «Neue Medien - Computer - Virtuelle Welten» die Diskussion eröffnet, welche Rolle moderne Technik und ihre medialen Ausprägungen für die hier diskutierten Medienbegriffe und in Folge für die unterschiedlichen Konzeptionen von Medienbildung spielen.

Zur Bestimmung des Medienbegriffs verweisen Meder, Sesink und Marotzki auf McLuhans Medientheorie. Das ist insofern interessant, als sich nur Meder intensiver damit beschäftigt (Meder 2004, 47), während sich die anderen auf kurze Umrisse und Referenzen auf McLuhan zur Verortung ihres Medienbegriffs beschränken. Ähnlich verhält es sich bei Meder und Spanhel mit dem Systembegriff Luhmanns. Spanhel verweist in der Begriffsbestimmung, aber auch in der Stellungnahme zu seinem Forschungszugang auf Luhmann (Spanhel 2002, 51; Spanhel 2006, 21 u. a.), während Meder sich mit dessen Theorie im Detail auseinandersetzt und diese begründend in seine bildungstheoretischen Ausführungen übernimmt (Meder 2001, 69). Bei Marotzki findet sich kein expliziter Medienbegriff, denn dieser geht in der strukturalen Analyse auf. (Jörissen und Marotzki 2008a, 57). Medien sind für ihn nicht Abstraktionen, sondern Konkretionen menschlichen Medienhandelns, welche er über den Begriff der Artikulation (Marotzki und Jörissen 2008a; 2009, 38; 2010, 27) definiert.

Neben oben referierten Gemeinsamkeiten lassen sich in den vier Konzeptionen von Medienbildung Unterschiede sowohl in den Definitionen, als auch darin auffinden, was sie mit ihrem Bestreben zur Begriffsdefinition bezwecken. So konnte beispielsweise aufgezeigt werden, dass Meder und Sesink sich zum Ziel setzen, eine pädagogische Medientheorie zu entwickeln. Dabei bezieht sich Meder auf die Sprachspieltheorie Wittgensteins (Meder 2004, 21) und arbeitet vor allem am Verständnis des Computers als Medium (Meder 2004, 97; 2015, 3ff), während Sesink Bildung ins Zentrum der medialen Entwicklung in der Moderne stellt (Sesink 2004, 147ff). Marotzki arbeitet hingegen eine Analysemethode heraus, um Bildungsprozesse nachzuvollziehen und in einem weiteren Schritt auch auf die Bedingungen der Möglichkeit von Bildung zu untersuchen (Marotzki 2004; 2007a). Spanhels Zugang und Ziel ist die Entwicklung einer Medienerziehung, für welche er keine eigenständige Medientheorie benötigt, sondern unterschiedliche Ansätze diskutiert, um den Begriff der Medien für seine Zwecke zu klären (Spanhel 2006, 61fff).

Eng mit den Begriffsbestimmungen zu «Medien» verbunden ist die Beziehung von «Sprache» und «Kultur» in ihrer Bedeutung für Bildung. Hier fanden sich Gemeinsamkeiten zwischen Meder und Marotzki, welche auch mit Spanhels Theorie in Beziehung gesetzt werden können. Medien stehen für Spanhel mit Kultur via Sprache im Austausch (Spanhel 2006, 69; 2005, 98; 2007, 40); ein Element, welches auch Meder aufgreift, wenn er auf Kultur als Differenz eingeht (Meder 2007, 63; 2011, 70). In Spanhels Verständnis ermöglicht Sprache als Meta-Medium Reflexion auf mediale Sinnerzeugung an welcher sie selbst beteiligt ist. (Spanhel 2003, 100; 2007,37). Meders Medientheorie basiert auf der Sprachlichkeit menschlicher Existenz (Meder 2007, 
63), jedoch entfaltet er eine eigenständige Theorie der «Sprachspiele» als Aushandlungsprozesse ${ }^{3}$. Marotzki bezieht sich nicht auf Sprache im Allgemeinen, sondern auf konkrete Artikulation ${ }^{4}$ (Marotzki und Jörissen 2008a, 58; Jörissen und Marotzki 2009, 38; Marotzki und Jörissen 2010, 27), als Ausprägungen medialer Darstellung in Bezug auf Bildungsprozesse. Die Verhandlung kulturell bedeutsamer Inhalte und die Selbstdarstellung finden - hier stimmt er mit den anderen drei Autoren überein medial vermittelt statt. Sprache als Mittel zur Meta-Kommunikation, welche im Sinne einer reflexiv auf die Lebensvollzüge gerichteten Bildung essentiell ist, findet sich folglich bei Meder, Marotzki und Spanhel. Zur Rolle der Sprache ist noch auf eine Differenz zwischen Meder und Sesink hinzuweisen, denn beide verweisen auf sprachliche Dimensionen der Technik, bzw. auf Sprachlichkeit der Technik. Dieses Verhältnis wird jedoch sehr unterschiedlich argumentiert. Meder beschreibt den Computer als sprachliches Medium, bzw. als auf Sprache reflektierendes Medium (Meder 2015, 4), während Sesink den sprachlichen Charakter der Technik anders fasst. Für inn ist Sprache das Medium des Geltungsanspruchs als «Begegnungsort von Subjekt und Objekt» (Sesink 2014, 35). Ähnlich wie bei Meder wird Sprache als Verweisungszusammenhang auf eine äussere (und damit nie ganz fassbare) Realität gesehen. ${ }^{5}$ Die Computersprache hat einen vorschreibenden Charakter und ist Ausdruck des Bestrebens nach Autonomie, also Unabhängigkeit von externen Imperativen. Der Bezug zur Welt wird über Nützlichkeit (wieder-)hergestellt.

Sprache als Ausdrucksform sowie als reflexives Moment konnte als ein zentraler Gedanke identifiziert werden. Alle vier Autoren gewahren kulturelle Veränderungen, die unter anderem durch neue mediale Formen vorangetrieben, oder sogar ausgelöst werden.

In Bezug auf «Neue Medien», bzw. «das Neue Medium» zeigten sich mehr Differenzen als Gemeinsamkeiten. Die Referenz auf Computer und Internet als Informations- und Kommunikationsmedien findet sich bei allen vier Autoren, allerdings in sehr unterschiedlicher Ausprägung. Spanhel diskutiert die Auswirkungen technischer Entwicklung für die Pädagogik (Spanhel 2006, 102fff). Seine Theorie der Medienerziehung ist ein Antwortversuch auf die vielfältigen Problemlagen, die er in der modernen Medienentwicklung diagnostiziert. Das Internet und seine vielfältigen - positiv konnotierten - Möglichkeiten spielen bei Meder und Marotzki eine bedeutende Rolle. Marotzki beschreibt das Internet als einen Raum, der neben Kommunikation auch in verstärktem Masse kulturelle, soziale, sowie gesellschaftliche Partizipation

3 Sprache als Raum repräsentiert alle möglichen Formen von Bedeutungen und durch die Verhandlung der innerhalb bestimmter Kulturen bedeutsamer Zeichen werden bestimmte Variationen in den Vordergrund gerückt. Sie nehmen Einfluss auf individuelle Sprachräume und deren Struktur. (Meder 2007, 61)

4 Sie finden auf drei Ebenen statt, nämlich der präreflexiven leiblichen, der narrativen und der metareflexiven. Sprache spielt einerseits als Ausdrucksmittel, andererseits in distanzierender Bezugnahme auf den medialen Ausdruck eine Rolle. (Jörissen und Marotzki 2009, 39)

5 Das heisst, die »natürlichen Sprachen« sind durch die Suche nach den richtigen Worten gekennzeichnet, Sesink stellt sie auch den formalen Sprachen gegenüber (Sesink 2014, 35). 
ermöglicht (Marotzki 2000, 255). Er reflektiert auf Chancen, die sich den Individuen durch neue Formen der Kommunikation und Informationsgewinnung - vor allem in Bezug auf die Mitgestaltung ihrer Lebenswelt und die eigene Identitätskonstruktion - bieten (Marotzki 20003, 137f; 2009, 153). Marotzki spezifiziert, anders als Spanhel, die Rolle der Pädagogik nicht als präventives, dekonditionierendes Eingreifen in den überfordernden Sozialisationsprozess, sondern richtet den Blick quasi immanent auf die Bildungsprozesse innerhalb der medialen Praxis (Marotzki 2000, 255).

Sesink stellt die Frage nach den Konsequenzen des modernen Medienwandels für das Menschenbild an sich (Sesink 2008a, 409). Für Meder steht die Grammatik der Sprachspiele im Zentrum, welche sich auch im Computer als sprachliches Medium zeigen lässt (Meder 2004, 165f). Beide Autoren diskutieren die Umkehrung der Zweck-Mittel-Relation. Meder definiert den Computer als «Problemlösungsautomaten» (Meder 2015, 3) für komplexe, sprachlich vermittelte Problemstellungen, die in der virtuellen Maschine wiederum sprachlich reflektiert und zurückgeworfen werden. Aus im virtuellen Prozess gefundenen Lösungen resultieren immer neue Problemlagen. Die Darstellbarkeit am Computer wird zum zentralen Gütekriterium der Fragestellungen: der Automat wird vom Mittel zur Problemlösung zum Zweck der Problemfindung erhoben. (Meder 2008b, 228) Sesink rekurriert auch auf die Idee des Computers als universelle Maschine, deren Anwendungsgebiete beinahe unerschöpflich scheinen, womit sich weniger die Frage nach den geeigneten Mitteln für bestimmte Handlungen und Prozesse stellt, als jene danach, wie diese Möglichkeiten ausgeschöpft werden können. Der Computer fordert somit geradezu die Suche nach Zwecken innerhalb der Räume an Möglichkeiten, die er selbst eröffnet und formt (Sesink 2008b, 15). An dieser Stelle wird eine Gemeinsamkeit mit Meders Problemlösemaschine offenkundig.

Wie gezeigt werden konnte, sind die Positionen der Autoren in Bezug auf «Neue Medien», «Computer» und «Virtuelle Welten» different, was zu einem regen Austausch und auch zu Kontroversen führt. Nichtsdestotrotz lassen sich auch in dieser Diskussion gemeinsame Schlüsselbegriffe auffinden, anhand derer der Diskurs darstellbar wird.

Durch die enge Verbindung zwischen Medien und Kultur lassen sich aus den verschiedenen Blickwinkeln Konsequenzen für die Pädagogik erahnen. Im nächsten Punkt sollen Referenzen auf den Bildungsbegriff diskutiert werden, um im Anschluss daran die Verbindungslinien der beiden Begriffskomplexe nachvollziehbar zu machen. 


\section{Bildungsbegriff}

Die Untersuchung des Bildungsbegriffs zeigt die gemeinsame Herkunft der vier Autoren auf. Alle vier definieren Bildung als Transformation des "Selbst- und Weltverhältnisses», was als gemeinsame Basis angesehen werden kann. Dabei beziehen sie sich auf Klafki (Spanhel 2006, 188), Humboldt (Meder 2011, 68), Kant (Marotzki 2004, 364, Sesink 2007a, 80) und Hegel (Sesink 2008b, 22). Als weiterer Schlüsselgedanke konnte die mediale Vermitteltheit von Bildung herausgearbeitet werden. In den vier Theorien wurden darüber hinaus «Bildungsideale, Visionen und Ziele», im Sinne normativer Zuschreibungen an den Bildungsbegriff, identifiziert.

Meder, Spanhel und Marotzki definieren das Bildungsverhältnis sehr ähnlich. Bei allen dreien kommt zusätzlich zum Selbst- und Weltverhältnis noch die mediale Dimension hinzu. Spanhel spricht von einer medial vermittelten «Erschließung von Mensch und Welt» (Spanhel 2006, 188), und auch Meder erweitert das für ihn dreifach Bildungsverhältnis - zu sich selbst, den anderen und den Sachen in der Welt - um dessen Vermittlung im Medium (Meder 2007, 67). Für Marotzki stellt sich das Bildungsverhältnis als Aufbau von Orientierungswissen dar (Marotzki 2004). Diese Form von Wissen verhält sich reflexiv zu neuen Informationen und integriert diese in das Selbst- und Weltverhältnis. Er beschreibt mithin den Bildungsprozess ähnlich wie Meder, wenn er die Aktualisierung des Selbst im Bildungsprozesses in den Mittelpunkt rückt (Marotzki 2007a, 132). Bei Sesink findet sich die Bezeichnung von Bildung als gestaltendes Verhältnis zu sich selbst und zur Welt (Sesink 2007b, 105) ebenso. Ausser dieser Konnotation weist seine Theorie aber keine Ähnlichkeiten mit den anderen auf, denn sein Bildungsverständnis leitet sich aus einer anderen theoretischen Bezugstheorie her. Sesink geht auf Kant und Hegel und den von den beiden in unterschiedlicher Ausprägung entwickelten Begriff der «Einbildungskraft»- wie er bei Žižek diskutiert wird - zurück (Sesink 2007a, 80ff; 2008b, 20ff). Dabei wird ein starkes Subjekt einer Welt der Objekte gegenübergestellt, welche es sich durch EinBildung zu Eigen macht. Bildung vollzieht sich im Modus der Aneignung und Kritik. Menschen sind in diesem Verständnis weniger im Modus eines Austauschs mit der Welt - oder im Bemühen um eine Harmonisierung des Verhältnisses, wie Spanhel meint - als in einem der Bemächtigung, Destruktion und Umformung nach der eigenen Ordnung. Das hat weitereichende Konsequenzen und führte unter anderem zur Entwicklung technischer Möglichkeiten in der Moderne (Sesink 2007b, 114). Dieser Gedanke einer in Auflösung befindlichen Welt taucht auch bei Meder und Marotzki auf, allerdings verstanden als Chance auf neue Bildungsmöglichkeiten. Auch Sesink versucht die positive Wende: Über die Idee der Wiedergutmachung (Sesink 2007a, 88) soll das destruktive Verhältnis wieder ins Lot gebracht werden.

Die Aussage «Bildung ist medial vermittelt» liesse sich an allen vier Theorien explizieren. Spanhel, Marotzki und Meder stellen Medien vermittelnd in den sozialen, bzw. kulturellen Prozess der Bildung. Bei Spanhel sind diese dann eine systemische 
Einheit im Kontext anderer Sinnsysteme, also auch z. B. der Erziehung. Zudem definiert er Medienbildung als in der Sprache als Metamedium - also Inhalte nicht nur vermittelndes, sondern ausserdem transzendierendes - realisiert. Bei Meder findet man Bildung als doppelte Reflexion im Bildungsraum sprachlicher Vermittlung. Aus der medialen Vermitteltheit von Bildung ist Sprache nicht wegzudenken, was auch bei Marotzki zum Ausdruck kommt. In der meta-reflexiven Zone der Artikulation also im Nach-Denken über einen Sachverhalt und seine Darstellung - ergibt sich die Relevanz für Bildungsprozesse. Diese sind für Meder und Marotzki durch Distanzierung im Sinne einer reflektierenden Bezugnahme gekennzeichnet. Zu Spanhel ergeben sich allerdings Differenzen, denn während dieser auf die Wechselbeziehungen von Bildung und Medien fokussiert, analysiert Marotzki einzelne Aspekte medialer Phänomene - speziell das Internet und Virtualität - bezüglich ihres Bildungswerts.

Aufgrund der Tatsache, dass Sesinks Mediendefinition schon von jenen Meders, Spanhels und Marotzkis abweicht, ist es naheliegend, dass auch in der Art und Weise, wie er eine Verbindung zwischen Bildung und ihrer medialen Vermittlung herstellt, ein Unterschied besteht. Das neue Medium als Möglichkeitsraum steht für ihn im Zentrum. Er ist gleichzeitig Ort der Verhandlung und Überschreitung. Folglich ist er im selben Moment Schauplatz von Bildung und ihr Produkt. Das Medium befindet sich für ihn nicht wie bei den anderen vier Autoren in einer vermittelnden Zwischenposition. Bildung ist für Sesink an sich mediale Vermittlung, weil sie selbst die Möglichkeiten schafft, sich Kritisch zur Welt zu verhalten und somit eine eigene Weltordnung zu schaffen. Damit ist sie im Medium verwirklicht. Ihr Ausdruck ist die selbstbestimmte Autorenschaft der Menschen.

Der Bildungsbegriff kommt bei allen vier Autoren nicht ohne eine Zielkategorie aus, was als normative Perspektive, also "Bildungsideale, Visionen und Ziele» aufgegriffen wird. Damit ist die die Formulierung eines Ideals oder eines Ziels des Bildungsprozesses in den analysierten theoretischen Konzeptionen gemeint. Meder, Spanhel und Marotzki beziehen sich jeweils auf Merkmale in Individuen, welche idealiter erreicht werden können, während Sesink das Ideal nicht in den sich bildenden Personen, sondern in den Voraussetzungen für Bildung im medialen Raum verortet.

Spanhel formuliert an sich eine «normative Ausrichtung der Medienerziehung» (Spanhel 2006, 183), welche sich einerseits an den «Normen und Werten der Gesellschaft» orientiert, andererseits an der Verhandlung ihrer eigenen Ziele als sogenannte «Pädagogische Identität» (Spanhel 2014, 142) mitwirkt. Medienbildung wird zusammengenommen als das Ergebnis von Mediensozialisation und Medienerziehung und der darin erworbenen Medienkompetenzen hin zu «moralischer und intellektueller Autonomie» mit dem generellen Ziel «Menschlicher Freiheit» (Spanhel 2006, 50f).

Meders Figur des «Sprachspielers» (Meder 2004, 157ff) ist - neben einer differenzierten Medientheorie - auch ein Bildungsideal. Im Unterschied zu Spanhel formuliert er keine Kompetenzen und auch keine Erziehungstheorie. Der Sprachspieler 
verkörpert jene Fähigkeiten, die in der Postmoderne gebraucht werden, wenn z.B. das homogene, ausgeglichene Subjekt aufbricht in ein Management von Teilidentitäten, oder Arbeitsprozesse via Rationalisierung in kleinste Teilchen zerlegt werden (Meder 2004, 170). Im Gegensatz zu Spanhel sind für Meder auch die gesellschaftlichen Normen und Werte Sprachspiele, die es zu durchschauen gilt.

In Anlehnung an den Modernediskurs beschäftigt sich auch Marotzki mit «krisentypen» (Jörissen und Marotzki 2009, 16). Sie bestehen in der Auflösung der gesellschaftlichen und kulturellen Verbindlichkeiten, die auch bei Meder und Spanhel eine Rolle spielen. Die Bildungsaufgabe ist der Umgang mit der Unbestimmtheit, welche über «Tentativität» (ebd., 19f) erreicht wird. So bezeichnet Marotzki die Fähigkeit, in unbekannten Situationen über Orientierungswissen handlungsfähig zu bleiben und die Leerstellen aktiv für die individuelle Entwicklung zu nutzen. In dieser «neuen Subjektivität» lassen sich Ähnlichkeiten zu Meders Sprachspieler erkennen.

Sesink unterscheidet zwischen drei Bildungsidealen: Erstens dem Ideal der Kritik, welches der Bildung immanent ist (Sesink 2014, 27) und durch seine zersetzende Kraft mitunter zur Bedrohung wird. Zweitens die vollkommene Einbildungskraft als Idee absoluter Autonomie, ein Ideal, das - wie Sesink selbst einräumt -nicht realisierbar ist (Sesink 2007a, 90). Als drittes formuliert er das Bildungsideal der poietischen Technik, die sich in die menschlichen Zusammenhänge wieder eingliedert, die Formalisierung durch ihre Nützlichkeit sowie ihren Lebensweltbezug rematerialisiert und darüber hinaus zum Fortschritt menschlicher Entwicklung beiträgt (Sesink 2004, 96f). Ihr Beitrag besteht im Raum, den die Technik für Bildung freigibt. Somit ist nicht ein erreichter Zustand, sondern die Eröffnung von Möglichkeiten das eigentliche Ideal. Mit Marotzki könnte eine konkrete Ausformung dieser räumlichen Möglichkeitsschaffung aufgezeigt werden, wenn er nämlich das Internet als Handlungsraum fasst, welchem die eigene Kritik und Überschreitung schon inhärent ist (Marotzki 2000, 255).

Neben allgemeine Charakterisierungen der Bildung als Verhältnis und dessen mediale Vermitteltheit tritt noch das gemeinsame Element der normativen Kategorie, welche ein Ideal oder Ziel formuliert, worauf die Bildung hin steuert/steuern soll. Nachdem nun dargestellt wurde, in welchen Horizonten der Medien- und Bildungsbegriff definiert wird, sollen in einem weiteren Schritt argumentative Verbindungen zwischen den beiden Begriffen erfasst und als letztes auch Spezifika von Medienbildung - im Sinne einheimischer Begriffe - identifiziert werden.

\section{Verbindungen zwischen Bildung und Medien}

In diesem Kapitel soll sowohl die in den vier Theorien betonte mediale Vermitteltheit von Bildung in den Fokus rücken, als auch Argumentationslinien kontrastierend vor dem Hintergrund der vier Medienbildungstheorien dargestellt werden. Dabei stehen 
die Rekontextualisierung der für die Analyse vereinzelten Begrifflichkeiten und die Bestimmungen von Medienbildung im Zentrum.

Für den systematischen Vergleich war es bereichernd, auf die Fragerichtungen, bzw. die unterschiedlichen theoretischen Hintergründe der Autoren zu blicken. Im Interesse der Rekontextualisierung wurden über die Fragestellung der Verbindung zwischen Bildung und Medien einzelne Aspekte herausgegriffen und auf Gemeinsamkeiten und Unterschiede mit ausgewählten Gesichtspunkten der Theorien der anderen Autoren eingegangen. Dabei wurden erstens die bei Spanhel, Meder und Marotzki aufgeworfenen Problematiken der Integration technischer Möglichkeiten in Lernprozessen beleuchtet und danach gefragt, welche Möglichkeiten und Konsequenzen die drei Autoren identifizieren. Auch die Aspekte «Medialer Raum» und «Mediale Sozialität» wurden beleuchtet. Obwohl die Diskussion dieser Verbindungen für die hier dargestellte Untersuchung sehr fruchtbar waren, sind im Zusammenhang dieses Artikels, welcher auf die Konstitutionsproblematik blickt und daher die zentralen Begrifflichkeiten im Fokus hat, die einzelnen Ausführungen in mancher Hinsicht in ihrer Darstellung zu umfangreich, und zudem in ihrer Detaildichte nicht darstellbar. Aus diesem Grund wird hier nur auf die Analyse in der Arbeit verwiesen.

Es ist im Anschluss an Gemeinsamkeiten und Unterschiede der Begriffsdefinitionen von «Bildung» und «Medien» und ihrer Verbindungen abschliessend die Frage zu klären, was Medienbildung nun im Sinne einer eigenständigen Begriffsdefinition ausmacht. Dabei wurde wieder die Frage gestellt, um welche Schlüsselbegriffe sich der Diskurs gruppiert und wie die Autoren dazu Position beziehen.

\section{Spezifika von Medienbildung}

Nachdem die Definitionen der beiden Komponenten des Begriffs, nämlich «Medien» und «Bildung» dargestellt und diskutiert wurden, soll nun darauf eingegangen werden, welche Schlüsselbegriffe als einheimische Begriffe des betrachteten Teildiskurses ausgemacht werden können. Aus den Bestimmungen von Medienbildung wurden drei zentrale Begriffe, nämlich «Kritik», «Autonomie» und «Handlungsfähigkeit», herausgegriffen und sollen je nach Theorie als Spezifikum von Bildung oder spezielle Bildung dargestellt werden.

\section{«Kritik»}

Der Begriff der Kritik ist für alle vier Autoren in ihrer Bestimmung des Bildungsbegriffs essentiell. Dabei verwenden nicht alle denselben Kritikbegriff. Es soll im Folgenden jedoch nicht um eine Diskussion der Kritikverständnisse gehen - was mit Sicherheit lohnend wäre - sondern darum, wie die Autoren Kritik in Beziehung zu Medienbildung positionieren. 
Spanhel sieht in der Sprache als Meta-Medium auch das kritische Moment aufgehoben, welches die reflektierende Bezugnahme zu sich selbst, zu den sozialen Beziehungen und zur eigenen Rolle ermöglicht und gleichzeitig Mittel zu ihrem Ausdruck ist (Spanhel 2006, 82). Für Meder bieten die Sprachspiele die Möglichkeit ihrer eigenen Überschreitung, welche in der sprachlichen Reflexivität angelegt ist. Der Sprachspieler als Figur der ästhetisch-kreativen Kritik (Meder 2004, 261) innerhalb der Grammatiken ist mitunter ein Kontrapunkt zum vernünftigen, ganzheitlichen Subjekt, wie es Spanhel andenkt.

Bei Sesink ist Kritik das Prinzip der Bildung. Sie zerlegt die Sprache in kleinste Teilchen, deren letzte Konsequenz Bits in der Eigenschaft von «Atomen der Kritik» (Sesink 2008b, 27) sind. Kritik in Abstraktion von der Lebenswelt und ihren Bezügen und Kritik der Kritik zur Restitution der essentiellen Elemente der alten Ordnung in die neue sind zentrale Momente der Medienbildung in der Moderne.

Marotzki betont die soziale Komponente von Kritik (Marotzki 2000, 255). Dies könnte eine Gemeinsamkeit mit Spanhel sein, der sich jedoch nicht so sehr auf Partizipation bezieht, sondern auf die distanzierende Gegenüberstellung von Individuum und Medienhandeln (Spanhel 2003, 100). Für Marotzki ist ein Zentraler Punkt, dass Kritik auch in jenem Medium stattfindet, dessen Strukturen reflektiert werden (Marotzki 2000, 255). Am Beispiel der Netzkritik exemplifiziert er, dass vor allem die partizipative Beschaffenheit des Internet die Prüfung innewohnender Geltungsansprüche möglich macht. Die kreative Komponente und immanente Kritik könnten mit jener der Sprachspielerfigur Meders verglichen werden.

Kritik als Grundmoment von Bildung findet sich in Form des Hinterfragens von Gegebenheiten und der Reflexion hegemonialer Strukturen als ihre wichtigen Momente. Dies führt zur Frage nach der Rolle der Heranwachsenden im Bildungsprozess, welche im Folgenden über den Begriff der Autonomie diskutiert werden soll.

\section{«Autonomie»}

Autonomie ist ein weitläufiger Begriff welcher, wie zu zeigen sein wird, zugleich grosse Bedeutung für Bildung - und damit Medienbildung - aufweist.

Als Erziehungsziel und Ausprägungen von Medienbildung benennt Spanhel moralische und intellektuelle Autonomie als selbstständiges, vernünftiges und kritisches (Medien-)Handeln sowie seine Reflexion (Spanhel 2006, 51). Kontrastierend dazu beschreibt Meder die Korrelation zwischen Zwang und Freiheit im Bildungsprozess als Spannungsfeld zwischen Ich und Welt (Meder 2007, 58). Freiheit - oder Autonomie - ist für ihn in den sozialen Kontext eingebettet und somit sprachlich bestimmt. Bildung bewegt sich in diesem sprachlichen Raum der Ermöglichung und Überschreitung der (medialen) Gegebenheiten (Meder 2011, 78f). Das Bildungsideal des Sprachspielers ist insofern autonom, als er mit den Sprachspielen selbst spielen kann und 
dadurch einen hohen Freiheitsgrad erreicht (Meder 2004, 260fff). Dabei wäre die von Spanhel betonte moralische Bildung selbst wieder ein Sprachspiel, welches es zu destruieren gälte.

Die Destruktionskraft von Sprache und darüber vermittelt von Bildung findet sich bei Sesink in ihrer schärfsten Ausprägung. In diesem Sinne ist Autonomie hier wörtlich gemeint und gleichzeitig als Erlösung aus der drohenden Auflösung. Einerseits ist die endgültige Autonomie in der leiblichen Welt nicht realisierbar, andererseits kann durch eine kritische Wendung auf die Kritik - und ein Innehalten - deren Zerstörungskraft verringert werden. Die Wiedergutmachung findet im Modus der Autonomie als neues Gesetz statt (Sesink 2004, 86). Medienbildung ist in diesem Zusammenhang die kritische Wendung, in Form von Reflexion auf die Entwicklung und die Rückbesinnung auf Weltbezüge im Kontrast zur Bemächtigung an der Natur. Im Vergleich zu Meders Figur des Sprachspielers müsste dieser also noch einen Schritt weiter gehen und nach der Destruktion und Neudefinition der Regeln sich auch mit der Nützlichkeit für die anderen Spielerinnen und Spieler auseinandersetzen.

Marotzki spricht in seiner Theorie der Medienbildung von «informationeller Selbstbestimmung» (Marotzki 2004b, 66), welche durch Individualisierung von Wissenskonstruktionsprozessen einerseits notwendig, andererseits möglich wird. Hier findet sich eine Vergleichbarkeit mit Spanhel, denn es geht um Wissen und darum, dass Medienbildung sich dazu in ein Verhältnis setzt. Marotzki bezieht sich auf Biographisierungsprozesse in der Moderne und betont, die Bedeutung sich in der sozial ausdifferenzierten, informationellen Welt zurechtfinden zu können. Trotz relativer Autonomie sieht Marotzki, ähnlich wie Meder, die Einzelnen zurückgebunden in ihre sozialen Kontexte. Für ihn ist dies auch, wie oben schon gezeigt wurde, im Bildungsprozess notwendig.

Autonomie verweist auf Eigenständigkeit, welche sich in Handlungen ausdrückt. Wie die Medienbildungstheorien im Einzelnen auf konkrete Handlungskontexte eingehen, soll Thema des nächsten Kapitels sein.

\section{«Handlungsfähigkeit»}

Medienbildung und Medienhandeln stehen in allen vier Konzepten in engem Zusammenhang, weshalb hier das Augenmerk auf diesem Aspekt von Medienbildung liegt.

Spanhel definiert Medienbildung über Verstehen, Erzeugen und Reflektieren medialer Botschaften, was die Handlungsdimension ins Zentrum stellt (Spanhel 2006, 58). Zusätzlich geht es ihm um eine aktive Beteiligung an der Entwicklung der Kultur über den Einsatz erworbener Medienkompetenzen. Medienerziehung überträgt somit die moralische und gesellschaftliche Aufgabe, in ihrem Hineinwachsen in die Gesellschaft, zusehends an die Heranwachsenden. 
Hier liegt ein Vergleichspunkt mit Meders Sprachspieler, dessen Bestreben, sich an Sprachspielen zu beteiligen, erst in ihrer Kritik und Dekonstruktion (Meder 2004, 259) aufgeht. Damit differenziert sich Meders Medienbildungsbegriff gegenüber Spanhels. Ein weiterer Handlungsbezug findet sich bei Meder in der realen Handlungsdimension der Virtualität im Kontext der Simulation. Während virtuelle (Spiel-)Welten für Spanhel mitunter bildungshinderlich sein können, sieht Meder in ihnen einen neuen Lernraum.

Meders Gedanke der sozialen Aushandlung kulturell bedeutsamer Sachverhalte - die Teilhabe am gesellschaftlichen Sprachspiel - findet sich auch bei Marotzki wieder. Medienhandeln hat zwei Komponenten, einerseits die Identitätskonstruktion, andererseits die Teilhabe an kulturellen Aushandlungsprozessen. Beide erhalten durch die neuen Formen von Sozialität im Internet neue Möglichkeiten und Risiken. Artikulation ist für Marotzki die konkrete mediale Handlung. In der Analyse konnten Parallelen zwischen Marotzkis Konzept der virtuellen Identitätskonstruktion und Meders Sprachspieler gefunden werden.

Die Poiesis-Theorie Sesinks stütz sich auf die Konkretion menschlicher Sinnvollzüge und verweist somit auf reales Handeln. Die hier interpretierbare Handlungsfähigkeit ergibt sich aus einer kritischen, auf die Kritik gerichteten Bewegung, die eigenständiges Handeln möglich macht.

Zusammenfassend kann gesagt werden, dass im Diskurs um Medienbildung «einheimische Begriffe» aufgefunden werden konnten. Diese in eine übergeordnete Konzeption der Medienbildung fassen zu wollen, würde den wissenschaftlichen Austausch, welcher die Disziplin, wie gezeigt werden konnte, lebendig hält, zum Verstummen bringen.

\section{Ausblick}

Fragt man nach der Konstitution einer wissenschaftlichen Disziplin, so sind Schlüsselbegriffe und zentrale Fragestellungen ebenso wichtig, wie der rege Austausch. Alle vier Autoren erörtern Phänomene moderner Medienentwicklung in Beziehung zum klassischen Bildungsbegriff und leiten daraus eine neue Medienbildung ab. Im Grunde prägen sie dabei keine neuen Begrifflichkeiten, sondern interpretieren schon bestehende im Kontext der Fragestellung, wie Pädagogik auf die Medienfrage reagieren könnte. Letztendlich geht es allen Autoren um die Frage, wie Handlungszusammenhänge beschrieben, angeregt und letztendlich zu einem bestimmten Ziel geführt werden können. Die Perspektive liegt darin, Möglichkeiten für Bildung aufzufinden und zugänglich zu machen. Dabei ist die (Weiter-)Entwicklung der ganzen Gesellschaft im Fokus. Medienbildung ist also nicht stille Beobachterin, denn ihre kritische Haltung hat Konsequenzen und richtet sich aktiv an Strukturen, deren Veränderung sie anregen möchte. Wie gezeigt werden konnte, ist die Eröffnung (neuer) Handlungsräume 
im medialen und damit kulturellen Raum eine der prägendsten Zielkategorien der vier Theorien.

Medienbildung steht vor einer Herausforderung, die für Bildung im Allgemeinen prägend ist: Sie soll, ohne in der Lage zu sein Prognosen zu stellen, auf potentielle Herausforderungen in der Zukunft vorbereiten. Als Ausgangspunkt dient den vier Autoren eine kritische Zeitdiagnose, aus welcher sie Vermutungen über eine mögliche Entwicklung ableiten. Der Status quo bietet aber keine sichere Basis mehr für Ausgangspunkte, wie sich in der Verhandelbarkeit der Prämissen, welche durch fluide Begrifflichkeiten geprägt sind, zeigte. Die Lösung ist bei Spanhel, Meder, Marotzki und Sesink, Unbestimmtheit als Ausgangspunkt festzulegen. Die daraus generierten Konsequenzen für Wissen, Lehren, Lernen und persönliche wie gesellschaftliche Entwicklung bilden die Basis für eine in die Zukunft und auf Handlungsfähigkeit gerichtete Medienpädagogik.

Der Umgang mit Information und Wissen ist in Zeiten des Internet eine Frage, die nicht nur im Alltag bedeutsam ist, sondern der Erziehungswissenschaft mit Blick auf die Tradierung des Wissens, die Formen des Lernens und deren kulturelle Bedeutung eine neue Richtung gibt. Dabei ist es nicht nur wichtig, wie Spanhel und Marotzki die neuen Wissensformen und Handlungsräume in ihrer praktischen Anwendung zu beforschen, sondern auch, wie alle vier Autoren der Analyse vorführen, sich um die Begrifflichkeiten und deren Bedeutungen anzunehmen.

Im Sinne der Grundlagenforschung einer Disziplin, die sich so nahe am Puls der Zeit bewegt und deren Konstitution damit unabgeschlossen bleiben muss, um die Nähe zu ihrem primären Forschungsobjekt nicht einzubüssen, ist eine distanzierende Betrachtung des Diskurses darum umso wichtiger.

\section{Literatur}

Fromme, Johannes, Stefan Iske, und Winfried Marotzki. Hrsg. 2011. Medialität und Realität. Zur konstitutiven Kraft der Medien. Medienbildung und Gesellschaft 16. Wiesbaden: VS Verlag für Sozialwissenschaften. doi:10. 1007/978-3-531-92896-8.

Fromme, Johannes, und Werner Sesink. 2008. «Einleitung». In Pädagogische Medientheorie, herausgegeben von Johannes Fromme und Werner Sesink, 7-11. Medienbildung und Gesellschaft 6. Wiesbaden: VS Verlag für Sozialwissenschaften. doi:10.1007/978-3-531-909714_1.

Grell, Petra, Winfried Marotzki und Heidi Schelhowe. Hrsg. 2010. Neue digitale Kultur- und Bildungsräume. Wiesbaden: VS Verlag für Sozialwissenschaften.

Gross, Friederike von, Winfried Marotzki, und Uwe Sander. Hrsg. 2008. Internet - Bildung - Gemeinschaft. Medienbildung und Gesellschaft 1. Wiesbaden: VS Verlag für Sozialwissenschaften. doi:10.1007\%2F97 8-3-531-90793-2. 
Jörissen, Benjamin. 2011. «Medienbildung»- Begriffsverständnisse und -reichweiten. In: Medienbildung und Medienkompetenz. Beiträge zu Schlüsselbegriffen der Medienpädagogik, herausgegeben von Heinz Moser, Petra Grell und Horst Niesyto, 211-234. München: kopaed. http://www.medienpaed.com/article/view/402.

Jörissen, Benjamin, und Winfried Marotzki. 2009. Medienbildung - eine Einführung. Theorie Methoden - Analysen. Bad Heilbrunn: Julius Klinkhardt.

Klafki, Wolfgang. 2001. «Hermeneutische Verfahren in der Erziehungswissenschaft». In Einführung in die pädagogische Hermeneutik, herausgegeben von Christian Rittelmeyer und Michael Parmentier, 125-147. Darmstadt: Wissenschaftliche Buchgesellschaft.

Marotzki, Winfried. 2009. «Bildung in virtuellen Welten». In Wie ist Bildung möglich?, herausgegeben von Lothar Wigger, 151-167. Bad Heilbrunn: Julius Klinkhardt.

Marotzki, Winfried. 2007. «Dimensionen der Medienbildung. Abschätzung der Reichweiten am Beispiel audiovisueller Formate (Film)». In Mit. Spieler. Überlegungen zu nachmodernen Sprachspielen in der Pädagogik. Norbert Meder zum 60. Geburtstag, herausgegeben von Hartwich, Dietmar, Christian Swertz und Monika Witsch, 127-140. Würzburg: Königshausen \& Neumann.

Marotzki, Winfried. 2004. «Bildung und Orientierung im Zeichen neuer Informationstechnologien oder: Warum Lara Croft eine kulturelle Ikone ist». In Kontinuität, Krise und Zukunft der Bildung. Analysen und Perspektiven, herausgegeben von Petra Korte. Münster: LIT.

Marotzki, Winfried. 2003. «Bildung und Internet». In Erziehungswissenschaftliche Zeitdiagnosen: Deutschland und Frankreich, herausgegeben von Jacky Beillerot und Christoph Wulf, 126-141. Münster: Waxmann.

Marotzki, Winfried. 2000. «Zukunftsdimensionen von Bildung im neuen öffentlichen Raum». In Zum Bildungswert des Internet, herausgegeben von Winfried Marotzki, Dorothee M. Meister und Uwe Sander, 233-258. Opladen: Leske \& Budrich.

Marotzki, Winfried, und Benjamin Jörissen. 2010. «Dimensionen strukturaler Medienbildung». In Medienkompetenz und Web 2.0, herausgegeben von Bardo Herzig, Dorothee M. Meister, Heinz Moser, Horst Niesyto und Stefan Aufenanger, 19-39. Jahrbuch Medienpädagogik 8. Wiesbaden: VS Verlag für Sozialwissenschaften. doi:10.1007/978-3-531-92135-8_2.

Marotzki, Winfried, und Benjamin Jörissen. 2008. «Wissen, Artikulation und Biographie: theoretische Aspekte einer strukturalen Medienbildung». In Pädagogische Medientheorie, herausgegeben von Johannes Fromme und Werner Sesink, 51-70. Medienbildung und Gesellschaft 6. Wiesbaden: VS Verlag für Sozialwissenschaften. doi:10.1007/978-3-531-909714_4.

Marotzki, Winfried, und Norbert Meder. 2014. Perspektiven der Medienbildung. Medienbildung und Gesellschaft 27. Wiesbaden: Springer. doi:10.1007/978-3-658-03529-7.

Meder, Norbert. 2015. «Neue Technologien und Erziehung/Bildung». Medienimpulse, Heft 1/2015: http://medienimpulse.at/articles/view/788. 
Meder, Norbert. 2011. «Von der Theorie der Medienpädagogik zu einer Theorie der Medienbildung». In Medialität und Realität. Zur konstitutiven Kraft der Medien, herausgegeben von Johannes Fromme, Stefan Iske und Winfried Marotzki, 67-81. Wiesbaden: VS Verlag für Sozialwissenschaften.

Meder, Norbert. 2008a. «Die Luhmannsche Systemtheorie und der Medienbegriff». In Pädagogische Medientheorie, herausgegeben von Johannes Fromme und Werner Sesink, 37-50. Wiesbaden: VS Verlag für Sozialwissenschaften. doi:10.1007/978-3-531-90971-4_3.

Meder, Norbert. 2008b. «Bildung und virtuellen Welten - Cyberbildung». In Internet - Bildung - Gemeinschaft, herausgegeben von Friederike von Gross, Winfried Marotzki und Uwe Sander, 227-239. Wiesbaden: VS Verlag für Sozialwissenschaften. doi:10.1007/978-3-53190793-2_11.

Meder, Norbert. 2007. «Theorie der Medienbildung. Selbstverständnis und Standortbestimmung der Medienpädagogik». In Jahrbuch Medienpädagogik 6. Medienpädagogik - Standortbestimmung einer erziehungswissenschaftlichen Disziplin, herausgegeben von Werner Sesink, 55-73. Wiesbaden: VS Verlag für Sozialwissenschaften. doi:10.1007/978-3-53190544-0.

Meder, Norbert. 2004. Der Sprachspieler: der postmoderne Mensch oder das Bildungsideal im Zeitalter der neuen Technologien. 2. überarbeitete Auflage. Würzburg: Königshausen und Neumann.

Rittelmeyer, Christian und Michael Parmentier. 2001. Einführung in die pädagogische Hermeneutik. Mit einem Beitrag von Wolfgang Klafki. Darmstadt: Wissenschaftliche Buchgesellschaft.

Schorb, Bernd. 2009: «Gebildet und kompetent. Medienbildung statt Medienkompetenz?». merz. Medien + Erziehung. Zeitschrift für Medienpädagogik, 50-56, 53. Jahrgang, Heft 5.

Sesink, Werner. 2014. «Eine kritische Bildungstheorie der Medien». In Perspektiven der Medienbildung, herausgegeben von Winfried Marotzki und Norbert Meder, 11-44. Wiesbaden: Springer. doi:10.1007/978-3-658-03529-7.

Sesink, Werner. 2008a. «Neue Medien». In Handbuch Medienpädagogik, herausgegeben von Uwe Sander, Friederike von Gross und Kai-Uwe Hugger, 407-414. Wiesbaden: VS Verlag für Sozialwissenschaften.

Sesink, Werner. 2008b. «Bildungstheorie und Medienpädagogik. Versuch eines Brückenschlags». In Pädagogische Medientheorie, herausgegeben von Johannes Fromme und Werner Sesink, 13-36. Wiesbaden: VS Verlag für Sozialwissenschaften. doi:10.1007/978-3-53190971-4_2.

Sesink, Werner. 2007a. «Bildung und Medium. Bildungstheoretische Spurensuche auf dem Felde der Medienpädagogik». In Jahrbuch Medienpädagogik 6. Medienpädagogik - Standortbestimmung einer erziehungswissenschaftlichen Disziplin, herausgegeben von Werner Sesink, Michael Kerres und Heinz Moser, 74-101. Wiesbaden: VS Verlag für Sozialwissenschaften. doi:10.1007/978-3-531-90544-0_4. 
Sesink, Werner. 2007b. «In höchster Auflösung: Das Abstraktionspotential der synthetischen Medien. Bildungstheoretische Überlegungen zur Emanzipation». In Medien - Macht - Gesellschaft, herausgegeben von Johannes Fromme und Burkhard Schäffer, 101-120. Wiesbaden: VS Verlag für Sozialwissenschaften.

Sesink, Werner. 2004. In-formatio. Die Einbildung des Computers. Beiträge zur Theorie der Bildung in der Informationsgesellschaft. Münster: LIT.

Sesink, Werner. 1990. «Künstliche Intelligenz), Systemreproduktion und Bildung». In Neue Sammlung, S. 193-207, 30. Jahrgang, Heft 2.

Sesink, Werner, Michael Kerres und Heinz Moser. Hrsg. 2007. Jahrbuch Medienpädagogik 6. Medienpädagogik - Standortbestimmung einer erziehungswissenschaftlichen Disziplin. Wiesbaden: VS Verlag für Sozialwissenschaften. doi:10.1007/978-3-531-90544-0.

Spanhel, Dieter. 2014. "Der Prozess der Medienbildung auf der Grundlage von Entwicklung, Lernen und Erziehung». In Perspektiven der Medienbildung, herausgegeben von Winfried Marotzki und Norbert Meder, 121-148. Wiesbaden: Springer. doi:10.1007/978-3-658-035297_6.

Spanhel, Dieter. 2007. «Zur Standortbestimmung der Medienpädagogik aus anthropologischer und bildungswissenschaftlicher Sicht». In Jahrbuch Medienpädagogik 6. Medienpädagogik - Standortbestimmung einer erziehungswissenschaftlichen Disziplin, herausgegeben von Werner Sesink, Michael Kerres und Heinz Moser 33-54. Wiesbaden: VS Verlag für Sozialwissenschaften. doi:10.1007/978-3-531-90544-0.

Spanhel, Dieter. 2006. Handbuch Medienpädagogik. Band 3. Medienerziehung. Handbuch Medienpädagogik in 5 Bänden, herausgegeben von Horst Dichanz, Bardo Herzig, Johannes Magenheim, Dieter Spanhel und Gerhard Tulodziecki. Stuttgart: KlettCotta.

Spanhel, Dieter. 2005. «Medienkompetenz nach PISA unter anthropologischen Aspekten». In Nach PISA. Konsequenzen für Schule und Lehrerbildung nach zwei Studien, herausgegeben von Volker Frederking, Hartmut Heller und Anette Scheunpflug, 96-111. Wiesbaden: VS Verlag für Sozialwissenschaften.

Spanhel, Dieter. 2003. «Die Bedeutung anthropologischer bzw. kulturanthropologischer Aspekte für die Medienpädagogik». In Homo medialis. Perspektiven und Probleme einer Anthropologie der Medien, herausgegeben von Manfred L. Pirner und Mathias Rath, 91-106. München: Kopaed.

Spanhel, Dieter. 2002. «Medienkompetenz als Schlüsselbegriff der Medienpädagogik?». In Medienkompetenz - Kritik einer populären Universalkonzeption. Forum Medienethik, 9. Jahrgang, Heft 1, 48-53. München: Kopaed. 